\title{
Isolation of MDCK cells with low expression of $m d r 1$ gene and their use in membrane permeability screening
}

\author{
ANA BOKULIĆ \\ JASNA PADOVAN* \\ DARIJA STUPIN-POLANČEC \\ ASTRID MILIĆ
}

Fidelta Ltd., 10000 Zagreb, Croatia

Accepted March 29, 2021

Published online April 2, 2021

\begin{abstract}
The Madin-Darby canine kidney (MDCK) cell line is frequently used for permeability screening in drug discovery. It contains endogenous transporters, most prominently canine multidrug resistance P-glycoprotein (Mdr1), which can interfere with studies of P-glycoprotein substrate assessment and permeability measurements. Because MDCK wild type (WT) is genetically heterogeneous, an isolation procedure was investigated in this study to obtain the subclonal line with low P-glycoprotein expression. The best clone obtained had up to 3-fold lower amprenavir efflux and P-glycoprotein expression in comparison to WT. Of 12 standard compounds tested that exhibited active efflux in WT cells, 11 showed a decrease in efflux in the isolated clone. However, the decrease was not below the cut-off value of 2 , indicating residual P-glycoprotein activity. Clone isolation via the limiting dilution method, combined with bidirectional amprenavir permeability for clone selection, successfully identified MDCK clones with substantially lower P-glycoprotein efflux and has been demonstrated as a useful tool for assessing passive permeability in early drug discovery.
\end{abstract}

Keywords: permeability, MDCK, efflux ratio, clone isolation, P-glycoprotein, qRT-PCR, LC-MS/MS

\section{INTRODUCTION}

The characterization of pharmacokinetic properties for new chemical entities in early drug discovery through various in vitro absorption/distribution/metabolism/excretion (ADME) and in vivo pharmacokinetic (PK) methodologies, is essential for the selection and progression of candidates into development. Compounds are characterized across an array of in vitro ADME assays, including the assessment/measurement of permeability, transporter involvement, solubility, metabolic stability, drug-drug interaction potential and protein binding. These assays are often optimized to screen large numbers of compounds and are continually further developed to increase capacity, robustness and predictability.

Since oral administration is the most desirable route of administration, due to production costs and simplicity of application, successful prediction of intestinal absorption is

\footnotetext{
*Correspondence; e-mail address: jasna.padovan@fidelta.eu
} 
one of the key moments in drug design with membrane permeability being the key property (1). Membrane permeability in drug discovery is regularly measured using in vitro non-cell (PAMPA) and cell-based assays using well-differentiated and polarized cell monolayers grown on a membrane filter that models the intestinal epithelia (2).

The usefulness of in vitro cellular permeability assays for the prediction of intestinal absorption has been demonstrated through numerous papers where authors aimed to describe the relationship between permeability coefficients and oral absorption and classify compounds correspondingly $(3,4)$. A recent publication by Newby et al. investigated permeability thresholds to distinguish between low, medium and highly absorbed compounds, using classification and regression tree (CART) statistical analysis on a large set of literature data. The authors confirmed a good correlation between fraction absorbed and permeability suggesting a carrier-mediated transport, metabolism and low solubility as the main reason for outliers (5).

The human epithelial colon adenocarcinoma (Caco-2) cell line was the first cell line to be extensively evaluated for the prediction of oral absorption, as it exhibits a well-differentiated brush border on the apical surface and tight junctions similar to enterocytes of the small intestine when cultured as a monolayer. In addition, as it expresses a variety of transporter proteins and metabolic enzymes, it is considered to be the gold standard. However, a long differentiation time of 21 days required for Caco-2, represents a major drawback for high-throughput screening, particularly during the early stages of discovery when a large number of compounds are screened.

Amongst alternative cell lines available, Madin-Darby canine kidney (MDCK) cells are frequently used in early drug discovery despite its nonhuman and nonintestinal origin, due to a significantly shorter differentiation time (3-4 days), robustness and good prediction of human intestinal absorption as confirmed in numerous studies $(5,6,7)$. On the grounds that MDCK cells express a relatively low number of transporters, they are a suitable recombinant model (8). Therefore, the MDCK clone with an over-expressed human P-glycoprotein (MDCKII-MDR1) is routinely used for assessment of permeability and involvement of the P-glycoprotein in the transport of compounds and consequently prediction of their intestinal absorption. P-glycoprotein (P-gp), also known as multidrug resistance protein 1 (MDR1), is an efflux pump identified on the surfaces of epithelial and endothelial cells including enterocytes. Because of its apical location and its broad substrate specificity, P-gp plays an important role in the pharmacokinetic properties of compounds, limiting the absorption of its substrates $(9,10)$.

MDCK cells express endogenous efflux transporters P-gp and multidrug resistance-associated protein 2 (Mrp2) at their apical membrane (11) with P-gp being the most prominent one (12). Since endogenous transporters can interfere with measured permeability, the FDA guideline proposes to measure the net-flux ratio which is the ratio of the efflux ratios (ER) of MDCKII-MDR1 and MDCK wild type (WT) cells (13). This approach has shown false negatives as canine P-gp has been shown to be present at different levels in WT and transfected cell line $(12,14,15)$. Moreover, a substrate for canine P-gp is not necessarily a substrate for human P-gp and vice versa. Additionally, MDCK is a heterogenetic line observable in subpopulations by various resistance and transport properties (16). Therefore, efforts have been made to overcome this drawback of MDCK cells. Di et al. reported an improved MDCK clone with low endogenous canine P-gp expression (MDCK-LE), obtained using fluorescence-activated cell sorting with flow cytometry (12). Thereafter, Simoff et al. 
produced MDCK cells with complete P-gp knockout using the breakthrough CRISPR-Cas9 technology (17). Besides the flow cytometry technique, single-cell isolation could be carried out with an array of different techniques, for example, laser capture microdissection or microcapillary aspiration (18). All these techniques require specific expertise and technologies. A simpler and applicable approach is the method of limiting dilution cloning, which uses extensive dilution of the cell suspension to deliver the single-cell colonies (19).

Based on these observations, our aim was to investigate the limiting dilution approach for the isolation and characterization of clones with low canine P-gp expression in MDCK cells. Such cells could diminish the rate of false results in transport studies as the impact of endogenous efflux would be reduced. Moreover, they would enable a simpler and straightforward determination of passive permeability and consequently improved the prediction of in vivo intestinal absorption. A set of selected compounds, ranging from low to high permeability, including P-gp and non-P-gp substrates, was tested in the isolated MDCK clone together with MDCK wild-type cells and the canine MDR1 knockout MDCKII cells.

\section{EXPERIMENTAL}

\section{Materials}

Madin-Darby canine kidney wild type (MDCK-WT) cells were obtained from Solvo Biotechnology (Hungary). MDCK knockout canine P-gp (MDCK-KO) was obtained from Sigma (USA) under 90 days license agreement. Dulbecco's Modified Eagle's Medium containing high glucose (DMEM), heat-inactivated fetal bovine serum (FBS), Glutamax-100, penicillin (10,000 $\left.\mathrm{U} \mathrm{mL}^{-1}\right)$-streptomycin $\left(10 \mathrm{mg} \mathrm{mL}^{-1}\right)$-amphotericin $\mathrm{B}\left(25 \mu \mathrm{g} \mathrm{mL}^{-1}\right)$, nonessential amino acid solution (NEAA), $0.05 \%$ Trypsin-EDTA, ethylenediaminetetraacetic acid (EDTA), Dulbecco's phosphate buffer saline (D-PBS), Lucifer yellow (LY), dimethylsulfoxide (DMSO), formic acid, liquid chromatography-tandem mass spectrometry (LCMS/MS) internal standard warfarin and the most of commercially available standard compounds were purchased from Sigma (USA). Acetonitrile (ACN) and methanol were purchased from Merck (Germany). Amprenavir was obtained from Moravek Biochemicals (USA). Millicell-24 and Millicell-96 cell culture inserts plates with $1 \mu \mathrm{m}$ pore size polyethylene terephthalate (PET) membrane were purchased from Millipore Sigma (USA).

\section{Cell culture}

MDCK cells were cultured under controlled atmosphere $\left(37^{\circ} \mathrm{C}, 95 \%\right.$ relative humidity, $5 \% \mathrm{CO}_{2}$ ) in DMEM that contained $10 \%$ FBS, $1 \%$ NEAA, $1 \%$ antibiotic/antimycotic and $1 \%$ glutamine. Media was removed from cell culture flask or dish (well) and cells were washed twice with EDTA saline $\left(0.7 \mathrm{~mL} \mathrm{~cm}^{-2}\right)$. After careful elimination of all remaining solutions, $20 \mu \mathrm{L} \mathrm{cm}{ }^{-2}$ of trypsin was added and incubated up to $10 \mathrm{~min}$ at $37^{\circ} \mathrm{C}$. To stop proteolysis, complete media $\left(0.7 \mathrm{~mL} \mathrm{~cm}^{-2}\right)$ was added to detached cells. Cells were counted using a hematology analyzer XT-2000i (Sysmex, Japan) and diluted in complete DMEM to a volume of $2.5 \times 10^{5}$ cells $\mathrm{mL}^{-1}$ for Millicell-96 well plates $(75 \mu \mathrm{L} /$ well $)$ or $3 \times 10^{5}$ cells $\mathrm{mL}^{-1}$ for Millicell-24 well plates ( $400 \mu \mathrm{L} /$ well). Cells were fed with fresh media $24 \mathrm{~h}$ post-seeding and used for permeability assay on days 3 or 4 . 


\section{Isolation of MDCK clones}

Clone isolation was done by the limiting dilution method. After four passages in culture, MDCK wild-type cells were seeded on 96-well flat-bottom plates (Greiner, Austria) as 0.3 cells per well. This was obtained by serial dilution in the master plate and further dilution in the incubation plate (Fig. 1). Ten incubation plates were prepared. After 14 days of incubation in the cell culture conditions, wells with clones were identified by a microscope Axiovert 135 (Zeiss, Germany). 102 clones were further transferred to 24-well plates and allowed to grow for 6 days. Thereafter, clones were expanded to 6-well plates. After 4 days of growing in 6-well plates, clones were harvested and cells were counted on XT-2000i (Sysmex, Japan). Cells were further divided into three parts: a sample for qRT-PCR, a cryopreservation vial, and the third aliquot was seeded on Millicell-96 for amprenavir permeability study (Fig. 2).

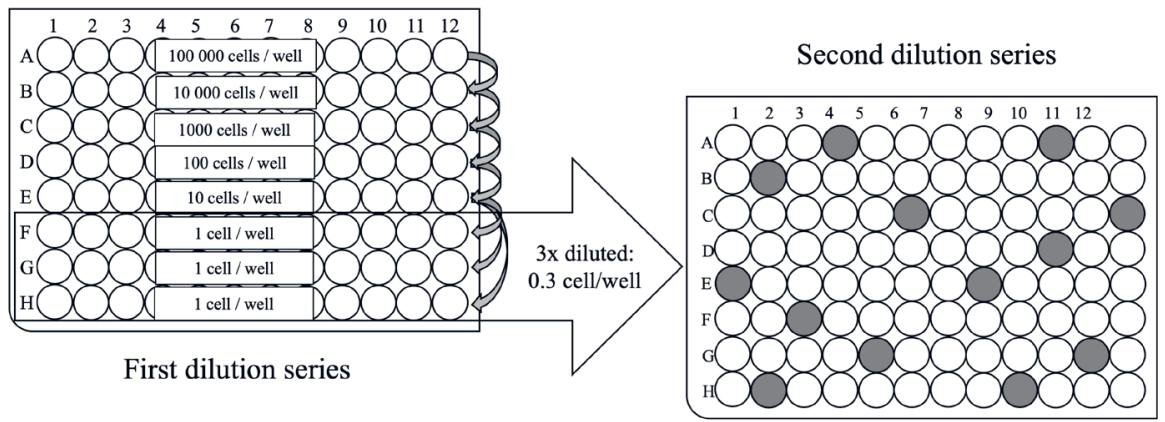

Fig. 1. Master plate and incubation plate layout: dilution steps to achieve seeding concentration of 0.3 cells per well.

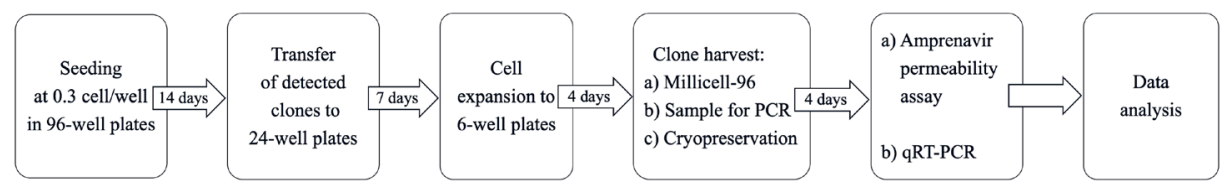

Fig. 2. Scheme of the clone isolation procedure.

Quantitation of transporter messenger $R N A$ level by real-time polymerase chain reaction (qRT-PCR)

The transporter messenger RNA (mRNA) levels in isolated clone cells were quantified according to the method by Livak et al. (20). Total RNA was isolated from the cells using RNeasy Mini Kit (Qiagen, Germany) and further converted to complementary DNA by High-Capacity cDNA Reverse Transcriptase Kit (Applied Biosystems, USA) according to the manufacturer's protocol. Expression of the canine Mdr1 transporter was analyzed by quantitative RT-PCR on 7300 Real-Time PCR system (Applied Biosystems, USA) using the TaqMan method and glyceraldehyde-3-phosphate dehydrogenase (Gapdh) as a house- 
Table I. Sequence of primers used for quantitative RT-PCR

\begin{tabular}{ccrc}
\hline $\begin{array}{c}\text { Protein } \\
\text { name }\end{array}$ & $\begin{array}{c}\text { Target } \\
\text { gene name }\end{array}$ & Sequence & $\begin{array}{c}\text { Forward (F)/ } \\
\text { Reverse (R)/ } \\
\text { Probe (P) }\end{array}$ \\
\hline $\begin{array}{c}\text { P-gp } \\
\text { (Mdr1), } \\
\text { dog }\end{array}$ & $\begin{array}{c}\text { abcb1 } \\
\text { (mdr1) }\end{array}$ & $\begin{array}{r}\text { 5'-CCACCACGATAGCTGAAAACATT-3' } \\
\text { 5'-CTTCCTTAACAGCTTTCTCAATCTCAT-3' }\end{array}$ & $\mathrm{F}$ \\
$\begin{array}{c}\text { Gapdh, } \\
\text { dog }\end{array}$ & Gapdh & 5'-CTATGGCCGCGAAAATGTCACCATG-3' & $\mathrm{P}$ \\
& & 5'-TGTCCCCACCCCCAATG-3' & $\mathrm{F}$ \\
\hline
\end{tabular}

-keeping gene. Primers and probes were designed in Primer Express software (Table I) and produced by Microsynth $(\mathrm{CH})$. Quantitative RT-PCR was performed at the following conditions: $2 \mathrm{~min}$ at $50{ }^{\circ} \mathrm{C}, 10 \mathrm{~min}$ at $95^{\circ} \mathrm{C}$, followed by 40 cycles of $15 \mathrm{~s}$ at $95^{\circ} \mathrm{C}$ and $1 \mathrm{~min}$ at $60^{\circ} \mathrm{C}$. Optimization of primer concentrations and primer validation for use with TaqMan Master Mix (USA) was performed according to the manufacturer's protocol. The reaction was performed using $100 \mathrm{nmol} \mathrm{L}^{-1}$ final concentration of both the primers and the probes. Each sample was analyzed in duplicates.

To quantify the expression, the comparative quantification method $\Delta \Delta \mathrm{CT}$ was used (Applied Biosystems). Obtained threshold cycle (CT) values were normalized to the house-keeping gene GAPDH $(\Delta \mathrm{CT})$, then further normalized to the WT sample (calibrator) $(\triangle \Delta \mathrm{CT})$. Finally, to determine the fold change in expression, $\Delta \Delta \mathrm{CT}$ was exponentially transformed $(2-\Delta \Delta \mathrm{CT})$ :

$$
\begin{gathered}
\mathrm{CT}_{\text {mdr1, sample }}-\mathrm{CT}_{\text {gapdh, sample }}=\Delta \mathrm{CT}_{\text {sample }} \\
\Delta \Delta \mathrm{CT}=\Delta \mathrm{CT}_{\text {sample }}-\Delta \mathrm{CT}_{\text {calibrator }} \\
\text { Fold difference }=2^{-\Delta \Delta \mathrm{CT}}
\end{gathered}
$$

\section{Bi-directional cell monolayer transport assay}

Cell monolayers were prepared for the permeability assay by washing and equilibrating for $30 \mathrm{~min}$ with a transport medium (D-PBS with $1 \%$ DMSO, v/v). To start the permeability assay, test compound solutions were added to donor wells and the transport medium to receiver wells. For the apical-to-basolateral direction, donor wells were apical compartments, while receiver wells were basolateral compartments and vice versa for the basolateral-to-apical direction. Volumes applied were based on the manufacturer's instructions: $400 \mu \mathrm{L}$ in the apical compartment and $800 \mu \mathrm{L}$ in the basolateral compartment. Test compounds were diluted in D-PBS to the final concentration of $10 \mu \mathrm{mol} \mathrm{L}^{-1}$, except for amprenavir $\left(0.5 \mu \mathrm{mol} \mathrm{L}^{-1}\right)$, vinorelbine $(1 \mu \mathrm{mol} \mathrm{L}-1)$, and fexofenadine, acebutolol, topotecan and rupintrivir at $5 \mu \mathrm{mol} \mathrm{L}{ }^{-1}$. The final DMSO content was $1 \%(v / v)$ as previously described in the literature $(21,22)$. Monolayers were incubated with a compound solution for $60 \mathrm{~min}$ at $37^{\circ} \mathrm{C}$ followed by sample collection for LC-MS/MS analysis.

Lucifer yellow at $100 \mu \mathrm{mol} \mathrm{L}^{-1}$ final concentration in compound solution, was used as a monolayer integrity marker. For each cell monolayer lucifer yellow permeability was measured using Infinite F500 reader (Tecan, $\mathrm{CH}$; excitation wavelength $485 \mathrm{~nm}$ and emission wavelength $530 \mathrm{~nm}$ ) and all wells with $P_{\text {app }}>3 \times 10^{-6} \mathrm{~cm} \mathrm{~s}^{-1}$ were discarded $(22,23)$. 


\section{LC-MS/MS analysis}

The LC-MS/MS analysis was performed on a Sciex API4000 triple quadrupole mass spectrometer (Applied Biosystems, USA) coupled to a Nexera ultra-high-performance liquid chromatography (UHPLC) (Shimadzu, Japan). A sample volume of $1 \mu \mathrm{L}$ was injected into a column and analytes were eluted using gradient chromatography (mobile phase A: $0.1 \%$ formic acid, mobile phase B: $0.1 \%$ formic acid in $90 \% \mathrm{ACN}$ and $10 \% \mathrm{H}_{2} \mathrm{O}$ ) at $50{ }^{\circ} \mathrm{C}$, with a flow rate of $0.7 \mathrm{~mL} \mathrm{~min}^{-1}$, leading to a total run time of 1.5-2 min. Digoxin was analyzed under basic conditions (mobile phase A: $10 \mathrm{mM}$ ammonium bicarbonate in $95 \%$ water and $5 \%$ methanol, mobile phase B: $100 \%$ ACN). Three different columns were used depending on the compound: Halo2 $2 \mu \mathrm{m} \mathrm{C18,20 \times 2.1} \mathrm{mm} \mathrm{(Advanced} \mathrm{Materials} \mathrm{Technology,} \mathrm{USA)} \mathrm{for}$ acebutolol, amprenavir, clarithromycin, diclofenac, digoxin, erythromycin, fexofenadine, prazosin, propranolol, ritonavir, rupintrivir and topotecan; Acquity UPLC $1.7 \mu \mathrm{m} \mathrm{BEH}$ $\mathrm{C} 18,50 \times 2.1 \mathrm{~mm}$ (Waters, USA) for atenolol, azithromycin, ranitidine and theophylline; and Luna Omega 1.6 $\mu \mathrm{m}$ Polar C18, 30 × $2.1 \mathrm{~mm}$ (Phenomenex, USA) for quinidine, vinblastine and vinorelbine. Positive ion mode with turbo spray, an ion source temperature of $550{ }^{\circ} \mathrm{C}$ and a dwell time of $150 \mathrm{~ms}$ were utilized for mass spectrometric detection. Quantitation was performed using multiple reaction monitoring (MRM) at the specific transitions for

Table II. MRM transitions of the standard compound set

\begin{tabular}{cc}
\hline Compound & MRM transition \\
\hline Acebutolol & $337.2 \rightarrow 116.2$ \\
Amprenavir & $506.2 \rightarrow 245.4$ \\
Atenolol & $267.2 \rightarrow 190.1$ \\
Azithromycin & $749.5 \rightarrow 591.2$ \\
Clarithromycin & $748.4 \rightarrow 158.1$ \\
Diclofenac & $296.1 \rightarrow 213.7$ \\
Digoxin & $798.5 \rightarrow 651.5$ \\
Erythromycin & $734.5 \rightarrow 158.1$ \\
Fexofenadine & $502.3 \rightarrow 466.3$ \\
Prazosin & $384.1 \rightarrow 95.1$ \\
Propranolol & $260.1 \rightarrow 182.8$ \\
Quinidine & $325.1 \rightarrow 172.1$ \\
Ranitidine & $315.2 \rightarrow 176.0$ \\
Ritonavir & $721.3 \rightarrow 296.1$ \\
Rupintrivir & $599.2 \rightarrow 345.2$ \\
Theophylline & $181.0 \rightarrow 124.3$ \\
Topotecan & $422.3 \rightarrow 377.1$ \\
Vinblastine & $406.3 \rightarrow 376.2$ \\
Vinorelbine & $779.4 \rightarrow 658.3$ \\
\hline
\end{tabular}


each compound (Table II). Analyte and internal standard peak areas were reviewed and quantitated using the DiscoveryQuant 3.0 software (Applied Biosystems, USA).

\section{Data analysis}

Quantitation was based on the ratio of peak area of the compound to that of the internal standard. Apparent permeability coefficient $\left(P_{\text {app }}\right)$ values were calculated according to the following equation:

$$
P_{\text {app }}=\frac{V_{\mathrm{A}} C_{\mathrm{A}} \times 10^{6}}{t A C_{0}}
$$

where $V_{\mathrm{A}}$ is acceptor volume in receiver well, $C_{\mathrm{A}}$ is a measured amount of compound in receiver well at time $t, t$ is incubation time (3600 s), $C_{0}$ is the initial compound concentration at time 0 minute and $A$ is surface area of the cell monolayer $\left(0.7 \mathrm{~cm}^{2}\right.$ for 24 -well plate and $0.11 \mathrm{~cm}^{2}$ for 96 -well plate). Compounds were classified as low permeable if $P_{\text {app }}<2 \times 10^{-6} \mathrm{~cm} \mathrm{~s}^{-1}$, and highly permeable when $P_{\text {app }}>10 \times 10^{-6} \mathrm{~cm} \mathrm{~s}^{-1}$.

The efflux ratio $(E R)$ was calculated using the equation:

$$
E R=\frac{P_{\text {app basolateral-to-apical }}}{P_{\text {app apical-to-basolateral }}}
$$

The quality of each $P_{\text {app }}$ value was evaluated through mass balance - the total amount of compound at the end of the experiment divided by the initial compound amount. Compounds with a mass balance of less than $70 \%$ and greater than $130 \%$ were excluded from the analysis, with an exception of propranolol for which mass balance in the apical-to-basolateral direction was between 60 and $70 \%$, but with $P_{\text {app }}$ values always in high range and the efflux ratio around 1.

All values are means of 1-3 experiments each performed in triplicate and the standard deviation (SD) expressed for values where $n=3$.

In order to assess the predictivity of RT-qPCR, receiver operating characteristic (ROC) curve analysis was generated, with the area under the curve (AUC) of RT-qPCR calculated (MS Excel).

\section{RESULTS AND DISCUSSION}

\section{Isolation of low efflux MDCK cells}

Using the limiting dilution method, a total of 102 clones were isolated from the MDCK-WT cell line and tested in quadruplicate for amprenavir efflux potential. Amprenavir was identified as a robust probe substrate for P-gp, as its transport across the MDCKII-hMDR1 cell monolayer depends only upon passive permeability and P-gp (24). In parallel, all clones were tested for P-gp expression by qRT-PCR to establish a relationship between amprenavir efflux and mRNA levels of P-gp. Although the overall linear correlation was weak, $R^{2}=0.46$, the highest and lowest points fitted well allowing clones to be ranked based on low canine Mdr1 expression and the low efflux ratio. ROC curve analysis was 


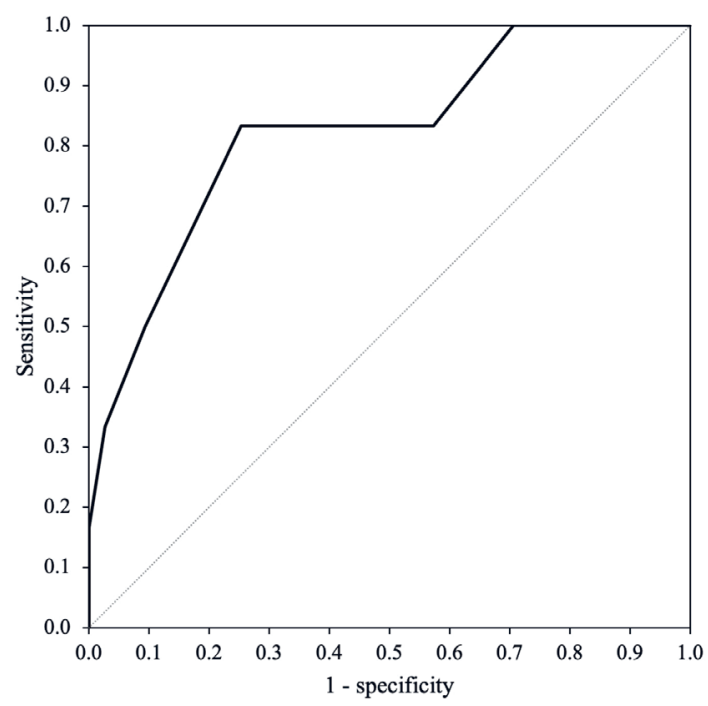

Fig. 3. ROC curve of low efflux MDCK clones detection by RT-qPCR. The thin diagonal line represents a completely uninformative test, wherein the area under the curve $(A U C)$ is 0.5 . The area under the ROC curve represented by the thicker line was 0.789 .

performed in order to evaluate the discriminatory ability to use RT-qPCR to aid the selection of clones with the lowest efflux ratio based on amprenavir as a control standard. The area under the ROC curve for RT-qPCR was moderate 0.789 (Fig. 3), however, the high specificity of RT-qPCR is accompanied by low sensitivity.

In addition to the best-ranked clone, clone 79, a few additional clones with moderate ranking (clones 69, 38, 1, 72 and 50) and one clone (clone 90) with the lowest ranking, were selected for further evaluation. In total, 7 clones were tested for efflux transporter activity

Table III. Efflux ratios of amprenavir, prazosin, topotecan and valsartan in clones selected for efflux transporter activity evaluation

\begin{tabular}{ccccc}
\hline Efflux ratio & Prazosin & Valsartan & Topotecan & Amprenavir \\
\hline Wild type & 1.3 & 0.5 & 4.0 & 6.8 \\
Clone 79 & 1.0 & 0.5 & 1.6 & 1.6 \\
Clone 69 & 1.1 & 0.4 & 2.5 & 3.0 \\
Clone 38 & 1.2 & 0.4 & 3.5 & 3.8 \\
Clone 1 & 1.3 & 0.4 & 2.1 & 4.1 \\
Clone 72 & 1.1 & 0.4 & 2.9 & 3.6 \\
Clone 50 & 1.3 & 0.4 & 2.1 & 3.0 \\
Clone 90 & 1.4 & 0.4 & 5.2 & 15.8 \\
\hline
\end{tabular}


(Table III). Prazosin and topotecan are known substrates of breast cancer resistant protein (BCRP) and P-gp, whereas valsartan is an MRP-2 substrate $(25,26)$. Prazosin and valsartan showed an efflux ratio below 2 indicating no involvement of active transport and no difference in the efflux ratio between clones. However, topotecan, a known BCRP but also a P-gp substrate, and amprenavir, a P-gp substrate, resulted in a reduced efflux ratio in clone 79 confirming lower expression of canine P-gp vs. the WT cells. In line with the qRT-PCR ranking, no difference in efflux ratio vs. the WT cells was observed for other clones. Therefore, clone 79 was chosen for further testing as it was characterized by the largest reduction in canine P-gp expression.

\section{Bi-directional transport across different cell lines}

A selected set of compounds was tested in the bi-directional transport assay across three cell lines: MDCK-79, MDCKII-WT, and the recently commercially available MDCK-KO. This set was selected to cover both high- and low-permeability, as well as non-MDR1 and MDR1 substrates with a wide range of efflux ratios in WT cells. The set included the following compounds: (a) atenolol representing low to moderate permeability and a non-P-gp substrate, (b) propranolol, theophylline and diclofenac as high permeability compounds and non-P-gp substrates, (c) fexofenadine, ranitidine, acebutolol, topotecan, erythromycin, clarithromycin, azithromycin, digoxin, ritonavir, rupintrivir, vinblastine, vinorelbine as low permeability compounds and P-gp substrates (d) prazosin, quinidine, amprenavir as moderate to high permeability compounds and P-gp substrates. The complete permeability results for tested compounds are available in Supplementary material.

Permeability results in WT MDCK cells were as expected for most compounds with the exception of fexofenadine, prazosin and ranitidine. In the current study, the permeability for ranitidine in both directions was low, resulting in an efflux ratio below 2 suggesting no P-gp involvement. Although reports in the literature have classified ranitidine as a P-gp substrate (27), other authors have reported efflux ratios below 2 (12, 28). A similar result was previously described in Caco-2 cells by Lentz et al., suggesting that for the low passive permeability drug ranitidine, P-gp may be less effective in limiting transport since low intracellular concentrations may not manifest an ER above 1.0 as small drug amounts are effluxed (29). This study further concluded that moderate passive permeability was necessary for P-gp to reduce the apical-to-basolateral permeability, which may also explain ER $<2$ observed for fexofenadine and prazosin. Fexofenadine was characterized by low bi-directional permeability and low efflux, although P-gp has been shown to be involved in the transport of fexofenadine $(30,31)$. On the contrary, prazosin is an example of an MDR1 substrate where the efflux ratio was most likely masked by a very high permeability in both the apical-to-basolateral and basolateral-to-apical directions in the standard experimental setting $(17,22)$.

The recent, commercially available, canine Mdr1 knockout MDCK cell line displayed symmetrical transport across the cell monolayer for all P-gp positive compounds except vinblastine, vinorelbine, and rupintrivir. Simoff et al. reported a similar result for vinblastine exhibiting an efflux above 2 both in wild-type and cMDR1 KO CL2 cells (17). The speculation was made that this could be attributed to the transport by different endogenous transporters such as cMrp2.

The isolated MDCK clone 79 with significantly lower amprenavir efflux activity compared to WT cells, was tested for bidirectional transport for a set of compounds. For P-gp 


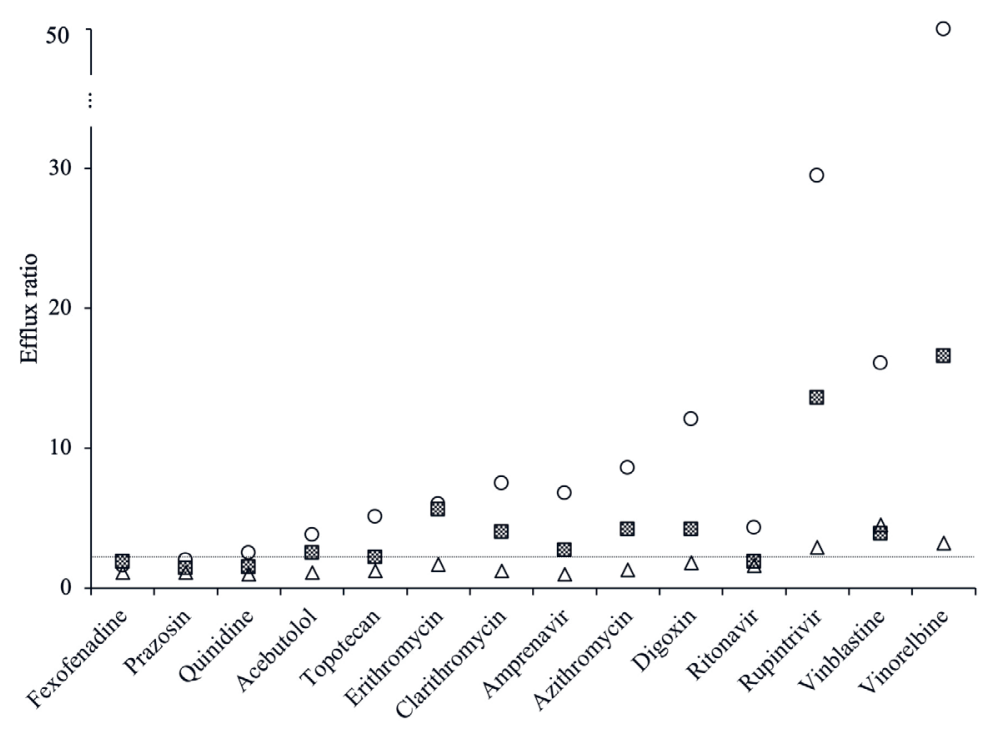

Fig. 4. Transport of known P-gp substrates in the MDCK-WT, MDCK-79, and MDCK-KO cell monolayer. The dashed line is set at the efflux ratio (ER) cutoff value of 2. ER above this value displays the involvement of active transport.

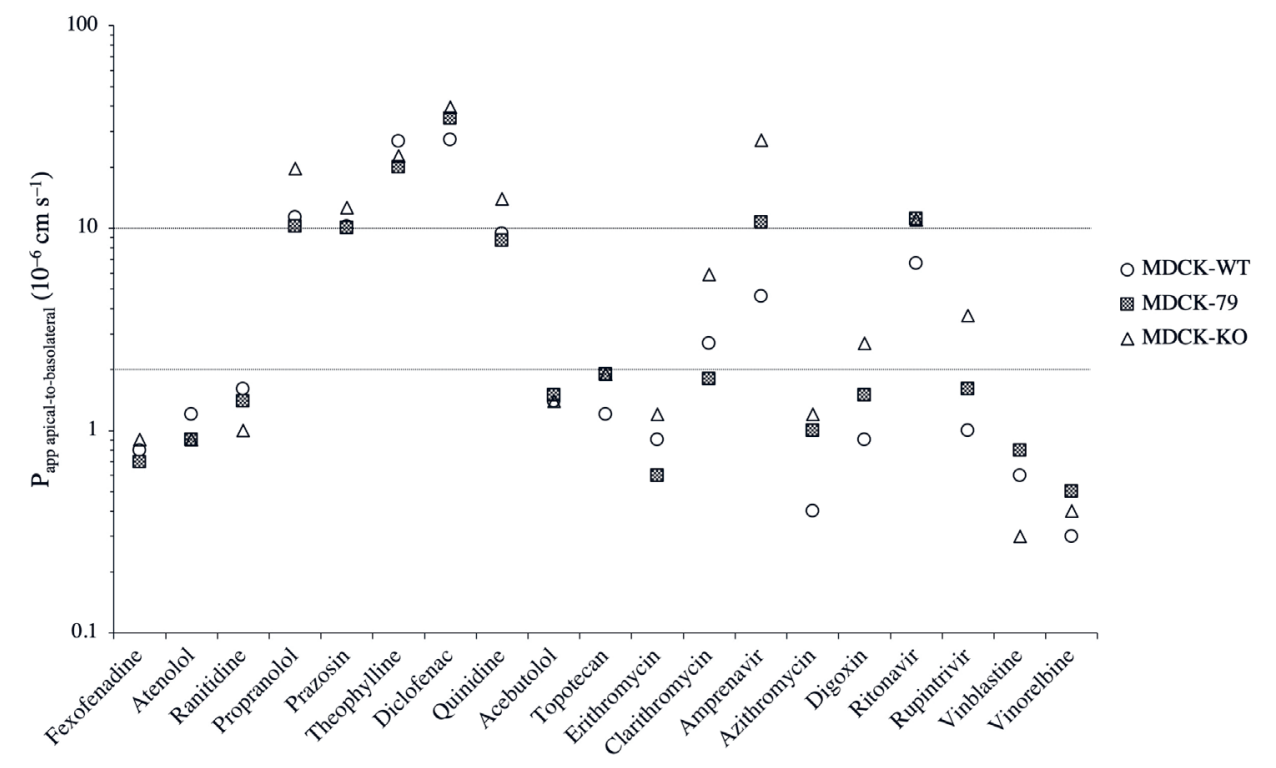

Fig. 5. Comparison of apical-to-basolateral direction permeability values for MDCK-79, MDCK-KO, and MDCK-WT cell monolayer transport assays. Compounds can be classified as low permeable if $P_{\text {app }}<2 \times 10^{-6} \mathrm{~cm} \mathrm{~s}^{-1}$, and highly permeable when $P_{\text {app }}>10 \times 10^{-6} \mathrm{~cm} \mathrm{~s}^{-1}$. 
substrates the efflux ratio in clone 79 was an average of 2.3 -fold lower in comparison to the MDCKII-WT, ranging from 1.6 for quinidine to 16.6 for vinorelbine vs. 2.5 and 53.9, respectively (Fig. 4). For compounds with a high efflux ratio (ER > 10), the fold change was 3.3. Nevertheless, the majority of the compounds would still be characterized as P-gp substrates with an ER $>2$, indicating residual P-gp activity in clone 79. Interestingly, erythromycin showed no decrease in efflux ratio in comparison to the WT cells, suggesting that the efflux ratio and effect that P-gp may play in transport is not entirely linearly related to expression (12). In an additional study with P-gp inhibitor GF120918 (data not shown), this efflux ratio in clone 79 was diminished for erythromycin to unity. Erythromycin has been reported to give false negative identification as a P-gp substrate in MDR1-MDCK and MDCK-WT due to a high efflux ratio in WT cells and has shown poor in vitro permeability across other cell lines $(12,32,33)$.

A comparison of permeability values $\left(P_{\text {app apical-to-basolateral }}\right)$ between clone 79 , WT and KO, showed that the rank ordering was similar for most compounds including fexofenadine, atenolol, ranitidine, propranolol, prazosin, theophylline, diclofenac, acebutolol, topotecan, erythromycin, azithromycin, vinblastine, vinorelbine (Fig. 5). This would suggest that for compounds with low permeability, which are P-gp substrates (ER $>2$ ), the permeability is not increased by removing residual canine Mdr1 activity as observed in clone 79 and $\mathrm{KO}$ cells in comparison to WT cells. Such results may be a result of inherent physicochemical properties and the profiling in early drug discovery should be based on passive permeability as previously suggested by Chen et al. (14). For six P-gp substrates, namely quinidine, clarithromycin, amprenavir, digoxin, ritonavir, and rupintrivir, the apparent permeability was somewhat higher in $\mathrm{KO}$ cells in comparison to WT, consequently affecting the change of the ranking class. For this group of compounds, the influence of P-gp was evident from the change in apparent permeability and was also observed in clone 79 with the exception of quinidine and clarithromycin. $P_{\text {app apical-to-basolateral }}$ values of clone 79 correlated better with the KO cell line $\left(R^{2}=0.93\right)$ than with the WT $\left(R^{2}=0.89\right)$. In comparison to published data in MDCK-LE clones (12), a good correlation for $P_{\text {app apical-to-basolateral }}$ values in clone 79 is observed for atenolol ( $1.31 \pm 0.42$ clone LE vs. $0.9 \pm 0.3$ clone 79$)$, ranitidine ( $1.02 \pm 0.04$ clone LE vs. 1.4 \pm 0.4 clone 79$)$, propranolol ( $6.96 \pm 0.31$ clone LE vs. 10.2 clone 79$)$, theophylline ( $23.1 \pm 1.6$ clone LE vs. 20 clone 79$)$, prazosin ( $10.9 \pm 2.2$ clone LE vs. 10 clone 79$)$ and quinidine (12.6 \pm 2.7 clone LE vs. $8.7 \pm 0.4$ clone 79 ), although there is a substantial difference in the canine P-gp expression. Specifically, based on real-time PCR, Di et al. reported a 200-fold lower expression of mRNA in MDCK-LE compared to MDCK-WT, obtained using cell sorting, whereas clone 79 had a 3-fold lower expression and is comparable to expression levels presented by Gartzke and Fricker, who also used the cell sorting methodology $(12,34)$.

In this study, we have shown that the limited dilution method can be used to isolate MDCK clones with lower canine P-gp activity such as clone 79 which showed a lower level of P-gp activity in comparison to the parental cell line. Such a clone could be used as a surrogate for estimating passive permeability, in addition to other methods typically used in drug discovery such as the use of potent P-gp inhibitor GF120918 in MDCK-MDR1 cells or parallel artificial membrane permeability assay (PAMPA) $(7,35)$.

\section{CONCLUSIONS}

Limiting dilution as a clone isolation method, combined with bidirectional amprenavir permeability for clone selection, represents an alternative method for isolating MDCK 
populations with lower P-gp efflux. Taking into account the qRT-PCR and amprenavir efflux results, the overall number of clones isolated would need to be increased in order to further understand the lack of correlation. An improved correlation would allow clone selection solely based on amprenavir efflux and thereby simplify the preparation of such cells. In spite of this, MDCK cells with a lower expression of canine P-gp are useful for estimating passive permeability and P-gp substrate assessment. Moreover, employing such a cell line in a high throughput format would allow the screening of a larger number of new compounds during the early stages of drug discovery for the prediction of permeability.

\section{REFERENCES}

1. P. Li, Preclinical in vitro screening assays for drug-like properties, Drug Discov. Today Technol. 2 (2005) 179-185; https://doi.org/10.1016/j.ddtec.2005.05.024

2. D. A. Volpe, Drug-permeability and transporter assays in Caco-2 and MDCK cell lines, Future Med. Chem. 3 (2011) 2063-2077; https://doi.org/10.4155/fmc.11.149

3. P. Artursson and J. Karlsson, Correlation between oral drug absorption in humans and apparent drug permeability coefficients in human intestinal epithelial (Caco-2) cells, Biochem. Biophys. Res. Commun. 175 (1991) 880-885; https://doi.org/10.1016/0006-291X(91)91647-U

4. S. Yee, In vitro permeability across Caco-2 cells (colonic) can predict in vivo (small intestinal) absorptioninman-factor myth, Pharm.Res. 14(1997)763-766; https://doi.org/10.1023/a:1012102522787

5. D. Newby, A. A. Freitas and T. Ghafourian, Decision trees to characterise the roles of permeability and solubility on the prediction of oral absorption, Eur. J. Med. Chem. 90 (2015) 751-765; https://doi. org/10.1016/j.ejmech.2014.12.006

6. J. D. Irvine, L. Takahashi, K. Lockhart, J. Cheong, J. W. Tolan, H. E. Selick and J. R. Grove, MDCK (Madin-Darby Canine Kidney) Cells: A tool for membrane permeability screening, J. Pharm. Sci. 88 (1999) 28-33; https://doi.org/10.1021/js9803205

7. T. T. Tran, T. Gales, B. Maleeff, T. Aldinger, J. W. Polli, A. Ayrton, H. Ellens, J. Bentz and A. Mittal, Exact kinetic analysis of passive transport across a polarized confluent MDCK cell monolayer modeled as a single barrier, J. Pharm. Sci. 93 (2004) 2108-2123; https://doi.org/10.1002/jps.20105

8. Y. Quan, Y. Jin, T. N. Faria, C. A. Tilford, A. He, D. A. Wall, R. L. Smith and B. S. Vig, Expression profile of drug and nutrient absorption related genes in Madin-Darby Canine Kidney (MDCK) cells grown under differentiation conditions, Pharmaceutics 4 (2012) 314-333; https://doi. org/10.3390/pharmaceutics4020314

9. F. Sharom, The P-glycoprotein multidrug transporter, Essays Biochem. 50 (2011) 161-178; https://doi. org/10.1042/bse0500161

10. M. V. S. Varma, K. Sateesh and R. Panchagnula, Functional role of P-glycoprotein in limiting intestinal absorption of drugs: Contribution of passive permeability to P-glycoprotein mediated efflux transport, Mol. Pharmaceutics 2 (2005) 12-21, https://doi.org/10.1021/mp0499196

11. L.-B. Goh, K. J. Spears, D. Yao, A. Ayrton, P. Morgan, C. R. Wolf and T. Friedberg, Endogenous drug transporters in in vitro and in vivo models for the prediction of drug disposition in man, Biochem. Pharmacol. 64 (2002) 1569-1578.

12. L. Di, C. Whitney-Pickett, J. P. Umland, H. Zhang, X. Zhang, D. F. Gebhard, Y. Lai, J. J. Federico, R. E. Davidson, R. Smith, E. L. Reyner, C. Lee, B. Feng, C. Rotter, M. V. Varma, S. Kempshall, K. Fenner, A. F. El-Kattan, T. E. Liston and M. D. Troutman, Development of a new permeability assay using low-efflux MDCKII cells, J. Pharm. Sci. 100 (2011) 4974-4985; https://doi.org/10.1002/ jps. 22674 
13. Food and Drug Administration Center for Drug Evaluation and Research (FDA/CDER), In vitro metabolism- and transporter-mediated drug-drug interaction studies guidance for industry, October, 2017; https://www.fda.gov/media/108130/download; access date November 10, 2020.

14. E. C. Chen, F. Broccatelli, E. Plise, B. Chen, L. Liu, J. Cheong, S. Zhang, J. Jorski, K. Gaffney, K. K. Umemoto and L. Salphati, Evaluating the utility of canine Mdr1 knockout Madin-Darby Canine Kidney I cells in permeability screening and efflux substrate determination, Mol. Pharmaceutics 15 (2018) 5103-5113; https://doi.org/10.1021/acs.molpharmaceut.8b00688

15. K. Kuteykin-Teplyakov, C. Luna-Tortos, K. Ambroziak and W. Loscher, Differences in the expression of endogenous efflux transporters in MDR1-transfected versus wildtype cell lines affect Pglycoprotein mediated drug transport, Br. J. Pharmacol. 160 (2010) 1453-1463.

16. J. M. Arthur, The MDCK cell line is made up of populations of cells with diverse resistive and transport properties, Tissue Cell 32 (2000) 446-450.

17. I. Simoff, M. Karlgren, M. Backlund, A.-C. Lindström, F. Z. Gaugaz, P. Matsson and P. Artursson, Complete knockout of endogenous Mdr1 (Abcb1) in MDCK cells by CRISPR-Cas9, J. Pharm. Sci. 105 (2016) 1017-1021; https://doi.org/10.1016/S0022-3549(15)00171-9

18. J. R. Yaron, C. P. Ziegler, T. H. Tran, H. L. Glenn and D. R. A. Meldrum, Convenient, optimized pipeline for isolation, fluorescence microscopy and molecular analysis of live single cells, Biol. Proced. Online 16 (2014) 9; https://doi.org/10.1186/1480-9222-16-9

19. R. I. Freshney, Cloning and Selection, in Culture of Animal Cells: A Manual of Basic Technique and Specialized Applications, John Wiley \& Sons, Ltd., New York 2010, pp. 207-225.

20. K. J. Livak, S. J. Flood, J. Marmaro, W. Giusti, and K. Deetz, Oligonucleotides with fluorescent dyes at opposite ends provide a quenched probe system useful for detecting PCR product and nucleic acid hybridization, Genome Res. 4 (1995) 357-362; https://doi.org/10.1101/gr.4.6.357

21. I. Hubatsch, E. G. E. Ragnarsson, and P. Artursson, Determination of drug permeability and prediction of drug absorption in Caco-2 monolayers, Nat. Protoc. 2 (2007) 2111-2119; https://doi. org/10.1038/nprot.2007.303

22. R. Mukkavilli, G. Jadhav and S. Vangala, Evaluation of drug transport in MDCKII-wild type, MDCKII-MDR1, MDCKII-BCRP and Caco-2 cell lines, CPB 18 (2018) 1151-1158; https://doi.org/10. 2174/1389201019666180308091855

23. Sigma-Aldrich, Technical bulletin, CompoZr ADMET/Tox Cell Lines C2BBe1 BCRP Knockout and Wild Type Cell Lines 24 Well Assay Ready Plates, 2013; https://www.sigmaaldrich.com/content/dam/ sigma-aldrich/docs/Sigma/Bulletin/1/mtox1002pc24bul.pdf; access date November 10, 2020.

24. P. Acharya, M. P. O'Connor, J. W. Polli, A. Ayrton, H. Ellens and J. Bentz, Kinetic identification of membrane transporters that assist P-glycoprotein-mediated transport of digoxin and loperamide through a confluent monolayer of MDCKII-HMDR1 cells, Drug Metab. Dispos. 36 (2008) 452-460; https://doi.org/10.1124/dmd.107.017301

25. The international transporter consortium, membrane transporters in drug development, Nat. Rev. Drug Discov. 9 (2010) 215-236; https://doi.org/10.1038/nrd3028

26. J. Rautio, J. E. Humphreys, L. O. Webster, A. Balakrishnan, J. P. Keogh, J. R. Kunta, C. J. SerabjitSingh and J. W. Polli, In vitro P-glycoprotein inhibition assays for assessment of clinical drug interaction potential of new drug candidates: A recommendation for probe substrates, Drug Metab. Dispos. 34 (2006) 786-792; https://doi.org/10.1124/dmd.105.008615

27. M. Hacker, W. S. Messer and K. A. Bachmann, Pharmacology: Principles and Practice; Academic Press, San Diego 2009, pp. 176-177.

28. M. Karlgren, I. Simoff, M. Backlund, C. Wegler, M. Keiser, N. Handin, J. Müller, P. Lundquist, A.C. Jareborg, S. Oswald and P. Artursson, A CRISPR-Cas9 generated MDCK cell line expressing human MDR1 without endogenous canine MDR1 ( $A B C B 1)$ : An improved tool for drug efflux studies, J. Pharm. Sci. 106 (2017) 2909-2913; https://doi.org/10.1016/j.xphs.2017.04.018 
29. K. A. Lentz, J. W. Polli, S. A. Wring, J. E. Humphreys and J. A. Polli, Influence of passive permeability on apparent P-glycoprotein kinetics, Pharm. Res. 17 (2000) 1456-1460; https://doi. org/10.1023/A:1007692622216

30. E. Gundogdu, E. Karasulu, I. Gonzalez Alvarez and M. Bermejo Sanz, Assessment of fexofenadine hydrochloride permeability and dissolution with an anionic surfactant using Caco-2 cells, Pharmazie 66 (2011) 747-753; https://doi.org/10.1691/ph.2011.1054

31. M. Cvetkovic, B. Leake, M. F. Fromm, G. R. Wilkinson and R. B. Kim, OATP and P-glycoprotein transporters mediate the cellular uptake and excretion of fexofenadine, Drug Metab. Dispos. 27 (1999) 866-871.

32. A. Milić, V. B. Mihaljević, J. Ralić, A. Bokulić, D. Nožinić, B. Tavčar, B. Mildner, V. Munić, I. Malnar and J. Padovan, A comparison of in vitro ADME properties and pharmacokinetics of azithromycin and selected 15-membered ring macrolides in rodents, Eur. J. Drug. Metab. Pharmacokinet. 39 (2014) 263-276; https://doi.org/10.1007/s13318-013-0155-8

33. J. Padovan, J. Ralić, V. Letfus, A. Milić and V. Bencetić Mihaljević, Investigating the barriers to bioavailability of macrolide antibiotics in the rat, Eur. J. Drug. Metab. Pharmacokinet. 37 (2012) 163171; https://doi.org/10.1007/s13318-011-0074-5

34. D. Gartzke and G. Fricker, Establishment of optimized MDCK cell lines for reliable efflux transport studies, J. Pharm. Sci. 103 (2014) 1298-1304; https://doi.org/10.1002/jps.23901

35. J. A. Ruell and A. Avdeef, Absorption Screening Using the PAMPA Approach, in Optimization in Drug Discovery (Ed. Z. Yan and G. W. Caldwell), Humana Press, Totowa 2004, pp. 37-64. 